\title{
Mental health innovations in Africa: lessons from Ethiopia and Zimbabwe
}

\author{
C. Lund ${ }^{1,2 *}$, Guest Editor \\ ${ }^{1}$ Department of Psychiatry and Mental Health, Alan J Flisher Centre for Public Mental Health, University of Cape Town, Cape Town, South Africa \\ ${ }^{2}$ Centre for Global Mental Health, Institute of Psychiatry, Psychology and Neuroscience, King's College London, London, UK
}

First published online 6 February 2017

During the last 5 years, mental health intervention research in low- and middle-income countries (LMIC) has been flourishing. This research has been spurred by substantial investments by funders such as the UK Department for International Development, Grand Challenges Canada, the National Institute of Mental Health, the European Commission and the Wellcome Trust. Many of these studies have been aligned with the research priorities identified by the Grand Challenges in Global Mental Health initiative (Collins et al. 2011). Key areas of investigation have included the delivery of evidence-based culturally appropriate and affordable mental health interventions in routine low-resource primary care and community settings, and the development of scale-able models of care. Efforts have been directed at making the clinical and economic arguments that will enable substantial investment from governments and development agencies, to narrow the treatment gap and improve the lives of people living with mental disorders in LMIC (Chisholm et al. 2016).

In parallel, there have been important policy commitments. In the World Health Assembly in 2013, 194 UN member states committed themselves to the four main objectives of the WHO Global Mental Health Action Plan (2013-2020), namely 'to strengthen effective leadership and governance for mental health; to provide comprehensive, integrated and responsive mental health and social care services in community settings; to implement strategies for promotion and prevention in mental health; and to strengthen information systems, evidence and research for mental health' (WHO, 2013). In a landmark high-level meeting titled: 'Out of the Shadows: Making mental health a global development priority' in Washington DC in April 2016, the President of the World Bank Dr Jim Yong Kim and the Director General of the WHO, Dr Margaret Chan committed themselves and their

* Address for correspondence: Prof C. Lund, Director, Department of Psychiatry and Mental Health, Alan J Flisher Centre for Public Mental Health, University of Cape Town, 46 Sawkins Road, Rondebosch 7700, Cape Town, South Africa.

(Email: crick.lund@uct.ac.za) organisations to give greater priority to the neglected area of mental health.

While these global commitments are important and laudable, they remain little more than pronouncements without real-world answers to the challenging 'how' questions: How do we integrate care for common mental disorders (CMD) in the context of an ongoing HIV pandemic in southern Africa? How do we address the complex needs of people living with severe mental illness in poor, low-resource settings? And how can we develop models of care that can be taken to scale while ensuring affordability, cultural acceptability and high-quality care?

In this issue of Epidemiology and Psychiatric Sciences, we present Editorials by two researchers at the forefront of addressing these questions. Dixon Chibanda and Charlotte Hanlon have both led highly innovative research to develop and scale up models of mental healthcare in sub-Saharan Africa: Chibanda in Zimbabwe and Hanlon in Ethiopia. Although their focus is on working in Africa, many of the lessons that they generate are relevant for work in other LMIC settings.

In his editorial, Dixon Chibanda describes the work of the Friendship Bench in primary care clinics in Harare, Zimbabwe (Chibanda, 2017). Originally developed by Chibanda and colleagues in 2006, the Friendship Bench consists of screening and assessment for CMD using a locally developed screening tool, the Shona Symptom Questionnaire (SSQ) (Patel et al. 1997). Primary care clinic attenders who score 8 or above on the SSQ are offered problem solving therapy, delivered by trained lay health workers. The intervention is delivered on benches situated in quiet and discrete areas within the grounds of primary care clinics in Harare. Chibanda and his colleagues have gone to great lengths to adapt an evidence-based psychological intervention - problem-solving therapy - to the local cultural setting, using local idioms of distress and recovery (Chibanda et al. 2016).

Chibanda describes the experience of taking the Friendship Bench intervention to scale in the city of Harare, now covering 70 primary care clinics, and delivered by over 300 lay health workers. He describes 
three major strategies that have enabled this scale-up. Firstly, community engagement has been vital to ensure the collaboration and buy-in of a wide range of stakeholders. Chibanda and colleagues coordinated a series of Theory of Change workshops, which enabled local stakeholders to contribute to the overall design of the programme, and to reach consensus on the outcomes and the pathways from programme initiation to impact. Secondly, evidence-based approaches have been essential, including not only evidence from randomised controlled trials (RCTs), but also evidence regarding how interventions can be delivered in routine low-resource settings. This included developing a good understanding of local human resources such as lay health workers, and drawing on their experience in the adaptation and delivery of the intervention. Thirdly, obtaining political buy-in from government, including early engagement and partnering with key policy makers in the City of Harare, has been essential for scaling up.

Such initiatives do not come without challenges, and Chibanda draws attention to three: ensuring fidelity and quality of care at scale; the need to expand the initiative to cover children and adolescents, and to develop common therapeutic approaches to address other conditions such as substance abuse and trauma; and broader political and economic volatility in Zimbabwe.

In her editorial, Charlotte Hanlon draws attention to the relative dearth of evidence from LMIC on tasksharing interventions for severe mental illness, compared with interventions for CMD such as depression and anxiety (Hanlon, 2017). She also points out some of the major challenges associated with conducting rigorous evaluations, such as RCTs, with people with severe mental illness in low-resource settings. These include the need for specialist providers, difficulties regarding capacity to consent to participate in studies or receive treatment, and the emphasis in many current research funding streams on primary care-based research.

Hanlon goes on to describe several recent initiatives to evaluate the real-world effectiveness of task-sharing models of care for severe mental illness in LMIC. These include the Programme for Improving Mental healthcare (PRIME) in Ethiopia, India, Nepal, South Africa and Uganda; the Task-Sharing for the Care of Severe mental disorders (TaSCS) trial in Ethiopia; the COllaborative Shared care to IMprove Psychosis Outcome (COSIMPO) trial in Nigeria and Ghana; the Rehabilitation Intervention for people with Schizophrenia in Ethiopia (RISE) trial; the BasicNeeds 'mental health and development model' in rural Kenya; the critical time intervention task sharing RedeAmericas trial in Brazil, Chile and Argentina; and the COmmunity care for People with Schizophrenia in India (COPSI) trial.

On the basis of these experiences, Hanlon outlines a way forward for research in this arena. She argues for the need to evaluate interventions using a 'full' task sharing model - required if we are to realistically narrow the massive treatment gap for severe mental illness in LMIC. This entails utilising primary care workers to diagnose, initiate treatment and provide ongoing care, under the training and supervision of specialists. Such approaches, she argues, will require major health system strengthening initiatives. These include developing chronic care approaches, empowering users and their families with information, engaging community health workers in adherence support, utilising mobile phone technologies for treatment support and health management information systems, and improving medication supply and utilisation. Adjacent to primary care approaches, additional resources are likely to be required for people with severe and complex problems, including specialist community-based care, livelihoods programmes, care in prisons and interventions to prevent homelessness and social complications associated with severe mental illness.

Several common elements can be identified from the experiences of Chibanda and Hanlon. Firstly, both conduct high-quality science in challenging lowresource settings, publishing their research in the leading international journals in their field. Secondly, both have undertaken thoughtful and innovative adaptations of evidence-based interventions, in a manner that goes beyond simply adapting and applying, but involves a substantial reworking of interventions to suit local cultural and health system settings. This has entailed extensive engagement with local stakeholders in the development, adoption and delivery of interventions. Thirdly, both have conducted policyrelevant research to develop and evaluate models that are affordable and scale-able.

These innovations provide (or will provide in the coming years) substantial evidence regarding how mental healthcare can be delivered in low-income countries and poor areas of middle-income countries. This includes evidence regarding cost, feasibility, acceptability, effectiveness and scale-ability. This is vital in the context of a dearth of substantial government investment in mental health, particularly in LMIC (WHO, 2015). Development assistance for mental health remains low, both in absolute terms and as a proportion of development assistance for health (Gilbert et al. 2015). As a community of mental health researchers, practitioners and advocates, it is vital that we continue to apply our research knowledge, and campaign for greater investment in mental 
healthcare, particularly for the millions who suffer unnecessarily in LMIC.

\section{Financial support}

This commentary is an output of the Programme for Improving Mental health carE (PRIME). The material has been funded by UK Aid from the UK Government; however, the views expressed do not necessarily reflect the UK Government's official policies.

\section{Conflict of Interest}

None.

\section{References}

Chibanda D (2017). Reducing the treatment gap for mental, neurological and substance use disorders in Africa: lessons from the Friendship Bench in Zimbabwe. Epidemiology and Psychiatric Sciences (This issue).

Chibanda D, Cowan F, Verhey R, Machando D, Abas M, Lund C (2016). Lay health workers' experience of delivering a problem solving therapy intervention for common mental disorders among people living with HIV: a qualitative study from Zimbabwe. Community Mental Health Journal

Chisholm D, Sweeny K, Sheehan P, Rasmussen B, Smit F, Cuijpers P, Saxena S (2016). Scaling up treatment of depression and anxiety: a global return on investment analysis. Lancet Psychiatry 3, 415-424.

Collins PY, Patel V, Joestl S, March D, Insel TR, Daar AS (2011). Grand challenges in global mental health. Nature 475, 27-30.

Gilbert BJ, Patel V, Farmer PE, Lu C (2015). Assessing development assistance for mental health in developing countries: 2007-2013. PLoS Medicine 12, e1001834.

Hanlon C (2017). Next steps for meeting the needs of people with severe mental illness in low- and middle-income countries. Epidemiology and Psychiatric Sciences (This issue).

Patel V, Simunyu E, Gwanzura F, Lewis G, Mann A (1997). The Shona Symptom Questionnaire: the development of an indigenous measure of common mental disorders in Harare. Acta Psychiatrica Scandinavica 95, 469-475.

WHO (2013). Mental Health Action Plan 2013-2020. WHO: Geneva.

WHO (2015). Mental Health Atlas 2014. WHO: Geneva. 